\title{
Generation and coherent detection of QPSK signal using a novel method of digital signal processing
}

\author{
Yuan Zhao*,, Bingliang $\mathrm{Hu}^{*}$, Zhen-An $\mathrm{He}^{*, \dagger, \S}$, Wenjia Xie ${ }^{\ddagger}$ and Xiaohui Gao* \\ * Key Laboratory of Spectral Imaging Technology, \\ $X i$ 'an Institute of Optics and Precision Mechanics, \\ Chinese Academy of Sciences, Xi'an 710119, China \\ $\dagger$ University of Chinese Academy of Sciences, \\ Beijing 100049, China \\ ${ }^{\ddagger} X i$ 'an Communication Institute, Xi'an 710106, China \\ $\S z y g g l 12345 @ 126 . c o m$
}

Received 6 December 2017

Accepted 10 January 2018

Published 6 February 2018

\begin{abstract}
We demonstrate an optical quadrature phase-shift keying (QPSK) signal transmitter and an optical receiver for demodulating optical QPSK signal with homodyne detection and digital signal processing (DSP). DSP on the homodyne detection scheme is employed without locking the phase of the local oscillator (LO). In this paper, we present an extracting one-dimensional array of down-sampling method for reducing unwanted samples of constellation diagram measurement. Such a novel scheme embodies the following major advantages over the other conventional optical QPSK signal detection methods. First, this homodyne detection scheme does not need strict requirement on LO in comparison with linear optical sampling, such as having a flat spectral density and phase over the spectral support of the source under test. Second, the LabVIEW software is directly used for recovering the QPSK signal constellation without employing complex DSP circuit. Third, this scheme is applicable to multilevel modulation formats such as $M$-ary PSK and quadrature amplitude modulation (QAM) or higher speed signals by making minor changes.
\end{abstract}

Keywords: Optical coherence; quadrature phase-shift keying; digital signal processing.

$\S$ Corresponding author. Present address: State Key Laboratory of Transient Optics and Photonics, Xi'an Institute of Optics and Precision Mechanics, Chinese Academy of Sciences, Xi'an 710119, China.

This is an Open Access article published by World Scientific Publishing Company. It is distributed under the terms of the Creative Commons Attribution 4.0 (CC-BY) License. Further distribution of this work is permitted, provided the original work is properly cited. 


\section{Introduction}

There has been a remarkable change in the area of high-speed optical fiber communications in recent years. Traditional binary ON-OFF signaling systems are being replaced with other more sophisticated modulation formats carrying more than one bit per symbol. ${ }^{1}$ Although mostly on-off keying (OOK) formats have been used in commercial applications so far, $M$-ary phase-shift keying ( $M$-PSK) and $M$-ary quadrature amplitude modulation ( $M$-QAM) have gained renewed attention to improve the spectral efficiency ${ }^{2}$ and meet the never-ending increasing

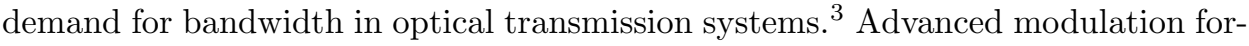
mats, such as differential quadrature phase-shift keying (DQPSK) ${ }^{4,5}$ and differential 8 -ary phase-shift keying (D8PSK), $\frac{6}{2}$ have already been demonstrated. Among various modulation formats that carry 2 bits of information per symbol, quadrature phase-shift keying (QPSK) is the most promising one because of its superior transmission characteristics. ${ }^{7,8}$ Several experiments have investigated the performance of QPSK systems with optical differential detection, where the receiver contains two sets of Mach-Zehnder interferometers and balanced photodetectors. $\frac{9}{}$ However, although synchronous detection of QPSK signals requires an SNR per bit of about $2 \mathrm{~dB}$ lower than differential detection, the synchronous coherent receiver needs either to use a local oscillator (LO) locked to the carrier phase or to recover the carrier phase after homodyne detection. In addition, the optical phase-locked loop (PLL) is still difficult to achieve because the practical voltage-controlled oscillator (VOL) operating at the optical stage is not available.

Coherent optical communication systems widely attract people's attention mainly because of their improved sensitivity over direct detection, their ability to receive complex modulation formats such as $n$-PSK and QAM as well as the ability to access the full information of the optical field in the electrical domain. $\frac{9}{}$ In Ref. 10 , the authors have suggested to utilize a digital implementation of a phased-locked loop for phase or even frequency fluctuation of LO laser as early as 1991. In fact, all of the recently published works ${ }^{11}-13$ focused on coherent receivers with employing digital signal processing (DSP) techniques for transmitter-local oscillator laser synchronization instead of using traditional optical phase-locked techniques. However, the sampling rate of the analog-to-digital convertors (ADCs) on the performance of QPSK signals was rarely studied.

Combining advanced modulation and coherent detection has been regarded as an emerging key technology for fulfilling the excepted bandwidth demands of future optical networks. In this paper, to retrieve the constellation diagram of the QPSK signals, we demonstrate an optical receiver that uses homodyne detection and DSP based on the LabVIEW software. In order to overcome the restriction on oversampling in the ADCs embedded within a LeCroy serial data analyzer (SDA; SDA 825Zi-A), we propose a novel extracting one-dimensional array of down-sampling method for reducing unwanted samples of constellation diagram measurement. 
This enables us to monitor, simultaneously, the amplitude and phase modulations of ultrafast optical QPSK signals.

\section{Principle}

Coherent detection performs the measurement of the electrical field of an optical QPSK signal by the interference with the local oscillation. The electric fields of the QPSK signal source under test and the LO are written as

$$
\begin{aligned}
\varepsilon_{D}(t) & =\sqrt{P_{D}^{*}} a_{\mathrm{IQM}}(t)^{*} \exp \left(j\left(\omega_{0} t+\varphi_{0}+\varphi_{\mathrm{IQM}}(t)\right)\right), \\
\varepsilon_{\mathrm{LO}}(t) & =\sqrt{P_{\mathrm{LO}}^{*}} \exp \left(j\left(\omega_{0} t+\varphi_{0}\right)\right),
\end{aligned}
$$

where $P_{D}, P_{\mathrm{LO}} a_{\mathrm{IQM}}(t)$ and $\varphi_{\mathrm{IQM}}(t)$ describe the signal source power, LO power, amplitude and phase modulations of the IQM, respectively. In this experiment, we implement a data-encoded optical source by modulation of a continuous wave (CW) laser and choose $w_{0}$ as the optical frequency of this CW laser. For LO source, the choice of its frequency is the same as the signal source because they come from the same CW laser. As depicted in Fig. 1, the source under test and the LO are coupled into the optical $90^{\circ}$-hybrid. The optical $90^{\circ}$-hybrid contains a pair of interferometers with a relative phase difference of $\pi / 2$. It outputs orthogonal quadratures (cosine and sine elements) to the $I$ - and $Q$-arms, respectively. The four outputs of the $90^{\circ}$-hybrid are received employing homodyne detection with two balanced photodetectors. Assuming an identical response $R(t)$ for the four detectors, the output signals of the two balanced photodetectors are

$$
\begin{aligned}
S_{A} & =\operatorname{real}\left[\exp \left[i\left(\varphi_{A}\right)\right] \cdot \int_{-\infty}^{+\infty} R(t) \cdot \varepsilon_{D}(t) \cdot \varepsilon_{\mathrm{LO}}^{*}(t) d t\right] \\
& =R(t) a_{\mathrm{IQM}}(t) \sqrt{P_{D} P_{\mathrm{LO}}} \cos \left(\varphi_{A}+\varphi_{\mathrm{IQM}}(t)\right), \\
S_{B} & =\operatorname{imag}\left[\exp \left[i\left(\varphi_{A}\right)\right] \cdot \int_{-\infty}^{+\infty} R(t) \cdot \varepsilon_{D}(t) \cdot \varepsilon_{\mathrm{LO}}^{*}(t) d t\right] \\
& =R(t) a_{\mathrm{IQM}}(t) \sqrt{P_{D} P_{\mathrm{LO}}} \sin \left(\varphi_{A}+\varphi_{\mathrm{IQM}}(t)\right), \\
S_{N} & =\exp \left[i\left(\varphi_{A}\right)\right] \cdot \int_{-\infty}^{+\infty} R(t) \cdot \varepsilon_{D}(t) \cdot \varepsilon_{\mathrm{LO}}^{*}(t) d t \\
& =R(t) a_{\mathrm{IQM}}(t) \sqrt{P_{D} P_{\mathrm{LO}}} \exp \left(\varphi_{A}+\varphi_{\mathrm{IQM}}(t)\right) .
\end{aligned}
$$

The electrical signals $S_{A}$ and $S_{B}$ contain information on the amplitude and the phase of the optical QPSK signal. The signals $S_{A}$ and $S_{B}$ are simultaneously sampled once after every symbol period $T$ with ADCs of the oscilloscope. However, for asynchronous sampling, the signal must be sampled at twice the symbol rate and then resampled to keep one sample per symbol. Therefore, the electrical ADCs require a sampling rate of

$$
f_{s, \mathrm{el}}=M_{\mathrm{el}} R_{s}=2 R_{s}
$$


where $R_{s}$ is the symbol rate of the received optical signal and $M_{\mathrm{el}}=2$ is the desired rational oversampling factor.

\section{Experimental Setup}

Our proposed photonic QPSK signal transmitter is indicated in Fig. 1. A CW laser is split into two paths using a coupler. The upper branch is connected to a DPMZM via a polarization controller for optical carrier modulation, and the lower branch is utilized as a local oscillation for homodyne coherent detection. A DPMZM contains two children Mach-Zehnder modulators (MZ-C1 and MZ-C2) nested within a third Mach-Zehnder modulator (MZ-P). There are three independent DC bias voltages and two RF inputs. $V_{\mathrm{RF} 1}$ and $V_{\mathrm{RF} 2}$ represent the RF modulating electrical voltages of MZM1 and MZM2. $V_{\text {bias1 }}, V_{\text {bias2 }}$ and $V_{\text {bias3 }}$ which are controlled by the bias voltage controller represent the DC bias voltages applied to MZ-C1, MZ-C2 and MZ-P, respectively. As illustrated in Fig. 1, the incoming light in the DPMZM is equally split into two arms, i.e. the in-phase $(I)$ and the quadrature $(Q)$ arms. The in-phase and quadrature components of the electric field are modulated independently by two pseudorandom binary sequences of length $2^{31}-1$, which are generated by a signal quality analyzer (MP 1800A). In both paths, a field amplitude modulator is achieved by operating the MZMs in the push-pull mode at the minimum transmission point. Furthermore, a relative phase shift of $\pi / 2$ in both paths can be adjusted by the bias voltage controller. The resulting NRZ-QPSK data are imprinted onto light from the $\mathrm{CW}$ laser at $1562 \mathrm{~nm}$ with a linewidth of $\sim 10 \mathrm{kHz}$ according to the manufacturer's specification. The choice of the NRZ formats stems from its experimental simplicity, since no pulse-carving stage is needed at the transmitter. Both the optical signal and the local oscillation are coupled using an optical $90^{\circ}$-hybrid. Then the in-phase and quadrature components of the optical QPSK signal are retrieved with two balanced photodiodes BPDA and BPDB, and converted to the electrical signals $I(t)_{\mathrm{BPDA}}$ and $I(t)_{\mathrm{BPDB}}$, respectively. The signals $I(t)_{\mathrm{BPDA}}\left(S_{A}\right)$ and $I(t)_{\mathrm{BPDB}}\left(S_{B}\right)$ are simultaneously sampled at a rate of 80 Gsamples/s with

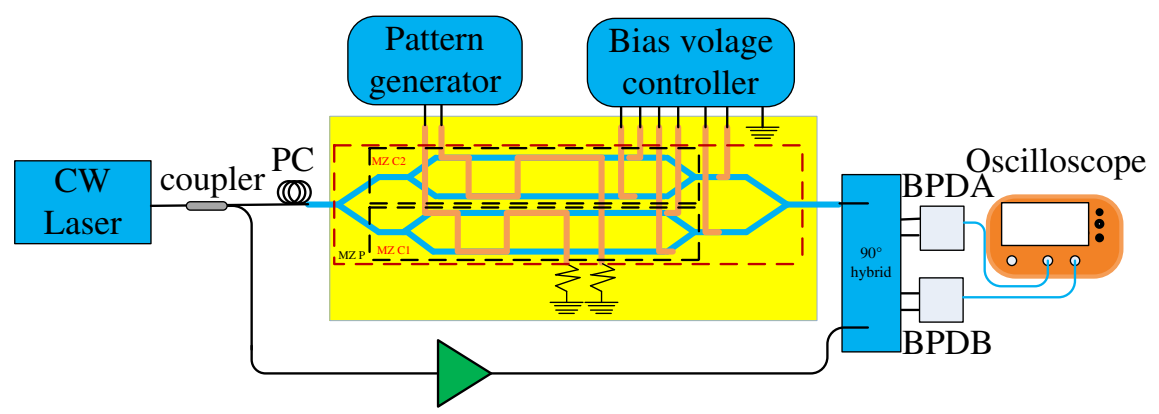

Fig. 1. Schematic of proposed photonic QPSK signal generator and homodyne demodulation (CW Laser: continuous wave laser, MZM: Mach-Zehnder modulator and PC: polarization controller). 
ADCs embedded within a LeCroy SDA oscilloscope. The sampled signals are then processed with the LabVIEW software. For all measurements, the output power of the EDFAs is adjusted to yield a signal power of $0 \mathrm{dBm}$ and an LO power of $6 \mathrm{dBm}$ at the input ports of the optical $90^{\circ}$-hybrids. The states of polarization of signal and LO are matched with manual polarization controllers.

\section{Signal Processing}

A block diagram of the used signal-processing algorithm for the configuration of homodyne detection is shown in Fig. 2. The output signals of the BPDA and BPDB are asynchronously acquired and digitized by a LeCroy SDA with two channels (LeCroy SDA 825Zi-A), a sampling rate of $80 \mathrm{~Gb} / \mathrm{s}$ per channel and $20 \mathrm{GHz}$ bandwidth. The acquired signals of the two channels of SDA are real and imaginary parts of the complex samples $I_{\mathrm{BPDA}}(n)+j I_{\mathrm{BPDB}}(n)$, where $n$ denotes the number of samples, and then we calculated and estimated the phase of the acquired samples using these complex samples. To improve the performance of this detection scheme, we establish a phase reference by taking more than one symbol into account, e.g. by averaging the phase over a number of consecutive symbols. The process of the phase estimation is as follows: the reconstructed signal samples $E(n)=\left[I_{\mathrm{BPDA}}(n)+j Q_{\mathrm{BPDB}}(n)\right]$ to the four power cancel the phase modulation $\Phi(=\pi / 4,3 \pi / 4,5 \pi / 4$ and $7 \pi / 4)$, since $E^{4} \infty \exp [j(4 \Phi)]$. The complex amplitudes

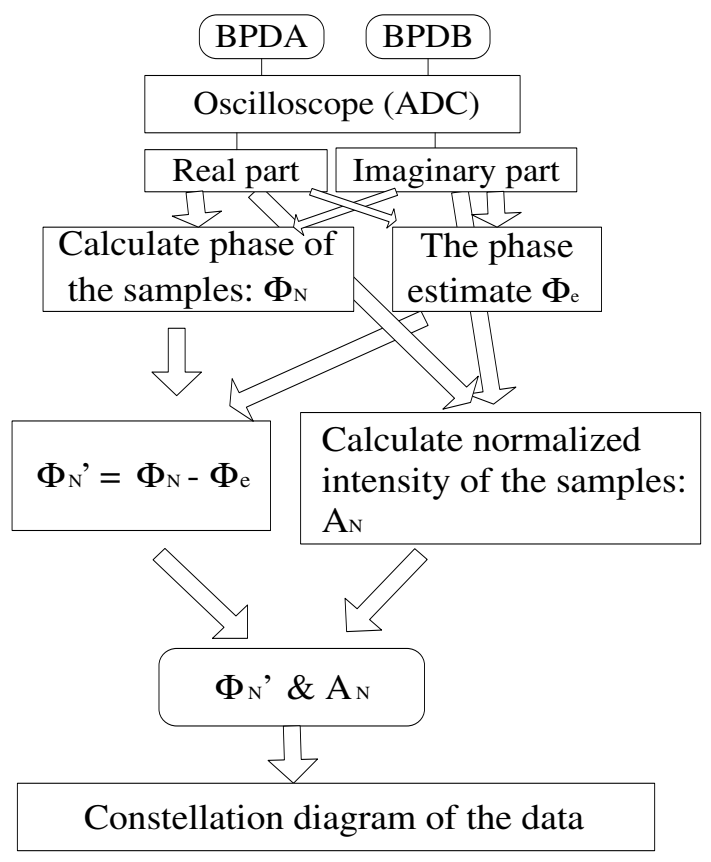

Fig. 2. A block diagram of the signal-processing algorithm. 
$E^{4}$ are summed, so that the phase is averaged over the entire block. The phase of the resulting complex amplitude is divided by 4 , leading to a phase estimation $\Phi$ given as

$$
\Phi=\frac{1}{4} \arg \left[\sum_{n=1}^{N} E^{4}(n)\right] .
$$

The resulting phase $\Phi_{n}^{\prime}=[\arg (E(i))-\Phi]$ and the normalized intensity $A_{n}$ of the $n$th sample are regarded as the phase and amplitude of reconstructed signal samples, respectively. Unfortunately, the employed SDA does not provide an option to externally clock its ADCs in order to set a desired sampling rate. Therefore, the received samples then need to be post-processed offline in a computer to recover the constellation diagram of the QPSK data signal.

\section{Results and Discussion}

We test the described algorithm in Fig. 2 using the measurement setup depicted in Fig. 1. A $\mathrm{LiNbO}_{3}$-based QPSK transmitter is used to generate an optical QPSK signal by using differentially precoded PRBS sequences with a word length of $2^{31}-1$ at a data rate of either $1 \mathrm{~Gb} / \mathrm{s}$ or $10 \mathrm{~Gb} / \mathrm{s}$. The representation of the samples $S_{N}$ can be made using a constellation diagram in the complex plane, which shows the amplitude and phase of the samples of the QPSK signals at a given position in the bit slot. For asynchronous sampling, the signal must be sampled at twice the symbol rate and then resampled to keep one sample per symbol. Therefore, the electrical ADCs require a sampling rate of

$$
f_{s, \mathrm{el}}=M_{\mathrm{el}} R_{s}=2 R_{s} .
$$

When the symbol rate of QPSK is 1 Gsymbols/s, the required electrical ADCs' frequency is $f_{s, \mathrm{el}}=2 R_{s}=2 \mathrm{GHz}$. Unfortunately, the ADCs embedded within a LeCroy SDA oscilloscope do not provide an option to externally clock them in order to set a desired sampling rate and its default sampling rate is 80 Gsamples/s. The leading edge and trailing edge of NRZ-QPSK signals are sampled since the sampling rate of SDA is 40 times higher than the required electrical ADCs' frequency. The samples for waveform diagrams are displayed in Figs. 3(a) and 3(b). There exist a lot of redundant samples since the sampling rate of ADCs is too high. These samples which can become a source of noise will display in the constellation diagram of QPSK signals and reduce the performance of the QPSK signals. Two different symbol rates of QPSK signals are used in order to validate this problem. The results are displayed in Fig. 3. Figures 3(a) and 3(b) display such waveform diagrams for the samples measured on $I$ - and $Q$-paths of 1 -Gsymbol/s NRZ-QPSK signal generated with an IQMZM. We discover that there exist several over-sampled samples in the waveform diagram of the reconstructed QPSK signal samples. Therefore, we must further down-sample the samples of the reconstructed QPSK signal. The array including the normalized intensity $A_{n}$ and the phase $\Phi_{n}^{\prime}$ is extracted for every 
waveform

(a)

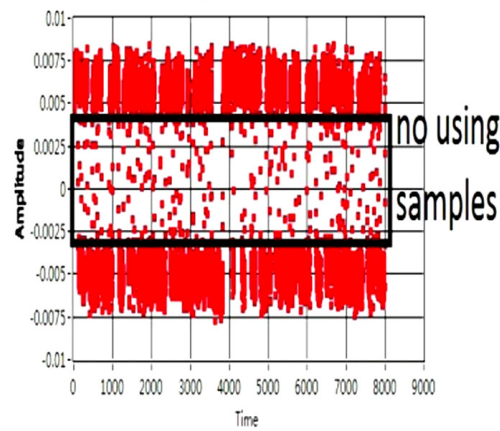

Constalletion diagram (C)

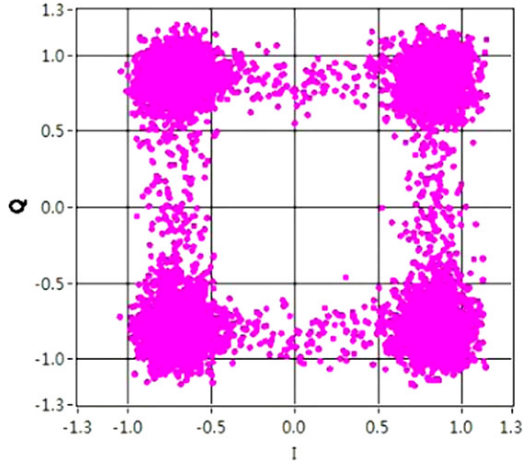

Constalletion diagram

(e)

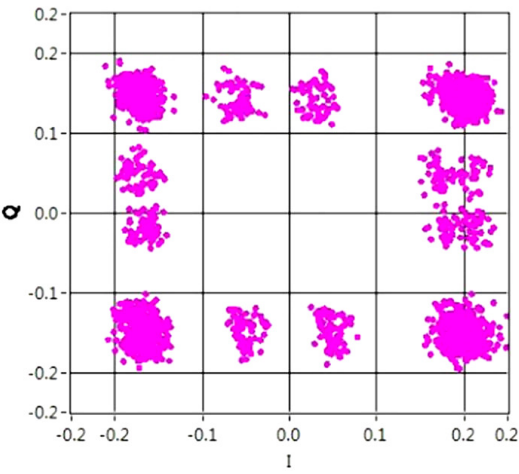

wavetorm 2

(b)

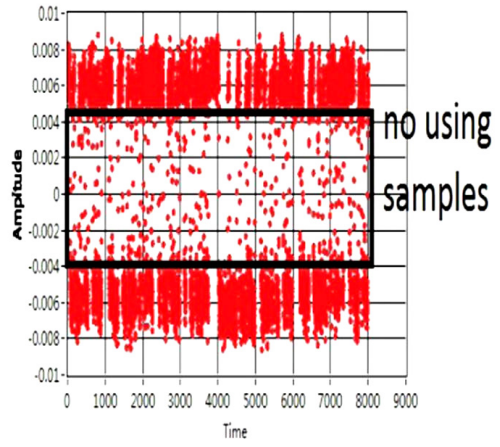

constellation diagram

(d)

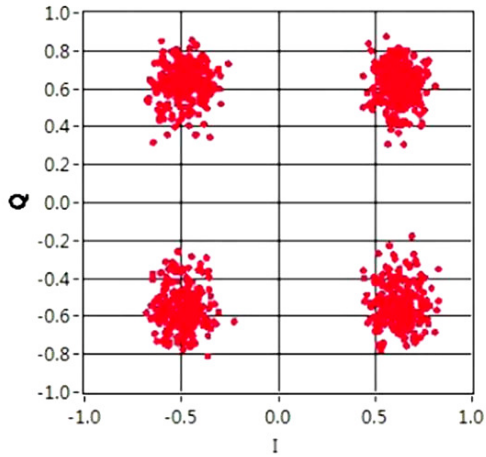

(f)

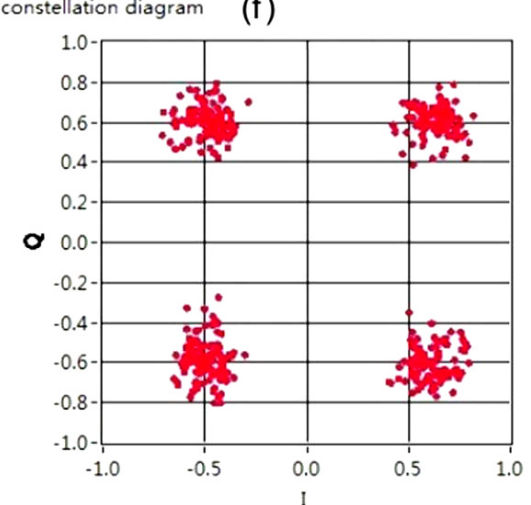

Fig. 3. (a) Waveform diagram for the samples measured on $I$-path of 1-Gsymbol/s NRZ-QPSK signal generated with an IQMZM. (b) Waveform diagram for the samples measured on $Q$-path of 1-Gsymbol/s NRZ-QPSK signal generated with an IQMZM. (c) The constellation diagram for the samples of 1-Gsymbol/s QPSK signal before extraction. (d) The constellation diagram for the samples of 1-Gsymbol/s QPSK signal after extraction. (e) The constellation diagram for the samples of 10-Gsymbol/s QPSK signal before extraction. (f) The constellation diagram for the samples of 10-Gsymbol/s QPSK signal after extraction. 
20 elements to ensure the required electrical ADCs' frequency by down-sampling the samples. Figures 3(c) and 3(d) display the constellation diagram for the samples of 1-Gsymbol/s QPSK signal before and after extraction. Figures 3(e) and 3(f) display the constellation diagrams for the samples of 10-Gsymbol/s QPSK signal before and after extraction. We find that the performance of the QPSK signal is enhanced by down-sampling of the samples. The amplitude and phase deviation are 0.131 and 1.718 , respectively.

\section{Conclusions}

We have demonstrated a simple and effective method to directly characterize the amplitude and phase of the QPSK signals in the constellation diagram. The constellation diagrams of the 1-Gsymbol/s and 10-Gsymbol/s QPSK signals are clearly displayed, showing that the performance of the QPSK signal is enhanced by using our DSP method.

\section{Acknowledgments}

This work was supported by Major Program of the National Natural Science Foundation of China (Grant No. 41530422) and the National Natural Science Foundation of China (Grant No. 11675258).

\section{References}

1. M. Skold, M. Westlund, H. Sunnerud and P. A. Andreson, J. Lightwave Technol. 27 (2009) 3662 .

2. C. Dorrer, C. R. Doerr, I. Kang, R. Ryf, J. Leuthold and P. Winzer, J. Lightwave Technol. 23 (2005) 178.

3. J. M. Gao, X. X. Xu, Q. J. Chang and Y. K. Su, Chin. Opt. Lett. 7 (2009) 109.

4. P. S. Cho, V. S. Grigoryan, Y. A. Godin, A. Salamon and Y. Achiam, IEEE Photonics Technol. Lett. 15 (2003) 473.

5. J. Gao, Q. Chang, T. Wang and Y. Su, Chin. Opt. Lett. 6 (2008) 550.

6. C. Kim, G. Goldfarb, Y. Han, Z. Li and G. Li, Opt. Express 13 (2005) 4044.

7. J. Kahn and K. P. Ho, IEEE J. Sel. Top. Quantum Electron. 10 (2004) 259.

8. D. S. Ly-Gagnon, S. Tsukamoto, K. Katoh and K. Kikuchi, J. Lightwave Technol. 24 (2006) 12.

9. R. Griffin and A. Carter, in Proc. Optical Fiber Communication Conf. and Exhibit (OFC) (2002), pp. 17-22.

10. A. Leven, N. Kaneda, U.-V. Koc and Y.-K. Chen, in Proc. Optical Fiber Communication and the National Fiber Optic Engineers Conf. (2007), pp. 1-3.

11. F. Derr, Electron. Lett. 23 (1991) 2177.

12. C. Dorrer, J. Lightwave Technol. 24 (2006) 313.

13. J. K. Fischer, R. Ludwig, L. Molle, C. Schmidt-Langhorst, C. C. Leonhardt, A. Matiss and C. Schubert, J. Lightwave Technol. 29 (2011) 378.

14. J. Pfeifle et al., Nat. Photonics 8 (2014) 375. 